\title{
Creating an Automated Health Attestation System During the COVID-19 Global Pandemic Using Google's G Suite
}

\author{
Erick M. Dubuque ${ }^{1}$ (I) $\cdot$ Brandon Franklin $^{2} \cdot$ Josh K. Pritchard ${ }^{3} \cdot$ Taylor Webb $^{1}$
}

Accepted: 13 December 2020 / Published online: 9 February 2021

(C) Association for Behavior Analysis International 2021

\begin{abstract}
As of October 2020, the COVID-19 global pandemic has infected over 40,000,000 people and has claimed over 1,000,000 lives globally (Johns Hopkins University, 2020). To mitigate the spread of the virus and their own liability, organizations have adopted multiple strategies to protect their employees and consumers. In addition to mask wearing, social distancing, and contact tracing, health attestations are being adopted by organizations that depend on physical contact between employees and consumers. The purpose of this tutorial is to describe how an automated health attestation and notification system can be created using Google's G Suite platform at little to no cost to the organization. When combined with other mitigation strategies and strong organizational policies, health attestations may be an effective component for organizations to include in their response to the global pandemic. The benefits and limitations of including health attestations as a component within an organization's COVID-19 policies are discussed.
\end{abstract}

Keywords COVID-19 $\cdot$ coronavirus $\cdot$ health attestations $\cdot$ Google G Suite $\cdot$ procedural safeguards

On March 11, 2020, the World Health Organization declared the COVID-19 outbreak a global pandemic (Hur \& Cheol Chang, 2020). At the time of this writing, COVID-19 has contributed to the deaths of over $1,000,000$ people globally and almost 250,000 in the United States alone (Johns Hopkins University, 2020). Nonessential businesses, schools, and many other entities have been forced to close or drastically alter their operational protocols to comply with governmentissued health mandates. Mass layoffs and furloughs have forced many workplaces to determine a way to keep employees safe while attempting to maintain productivity in the workplace. In the field of mental and behavioral health, the COVID-19 pandemic has interrupted services and created new challenges for professionals and businesses providing these services (Torales et al., 2020).

As health care businesses look to move forward, the adoption of mitigation strategies has become crucial. As explained

Erick M. Dubuque

erick.dubuque@louisville.edu

1 Department of Special Education, Early Childhood, and Prevention Science, University of Louisville, Louisville, KY, USA

2 Jefferson County Public Schools, Louisville, Kentucky, USA

3 Factari Holding, Inc., Orlando, FL, USA by Dubuque et al. (2020), many health care professions' codes of ethics contain language that ensures professionals and companies promote client well-being and provide ongoing treatment, services, and training. The COVID-19 pandemic brings unique challenges for these providers as they try to simultaneously provide services for their clients and mitigate the spread of the virus. Although mask wearing, testing, social distancing, handwashing, and contract tracing (Güner et al., 2020) take the forefront of many health protocols, health attestations can be another important mitigation strategy that businesses can implement to reduce the transmission of COVID-19.

Self-attestations and screenings are strategies that are already being used to monitor travel during the pandemic (Gostic et al., 2020). Although self-attestation screenings may miss some infected people due to a lack of symptoms at the time of screening, the accessibility and information offered still make it a valuable tool for businesses' mitigation strategies (Gostic et al., 2020; Jeong et al., 2020). When paired with proper hygienic behaviors, mask wearing, social distancing, and contact tracing (Güner et al., 2020), self-attestation screening tools can help reduce the risk and liability for health care professionals providing services by preventing those who are showing symptoms from coming into contact with those who are not currently symptomatic or infected (Figs. 1, 2 and 3).

Recently, Dubuque et al. (2020) shared a tutorial that described how to create an automated COVID-19 health 
attestation system using Microsoft Forms, Power Automate, and Outlook. The purpose of that tutorial was to help organizations mitigate the spread of COVID-19 and reduce liability if an employee or consumer became infected with the virus. The current tutorial attempts to achieve the same purpose by showing how a similar automated health attestation system can be created using the tools available through Google's G Suite.

G Suite contains multiple base applications and add-on programs. This tutorial requires the reader interact with three of them: Google Forms, Google Sheets, and Google Apps Script. The steps outlined are not the only way to set up an automated health attestation system in G Suite. There are addons (e.g., Boomerang for Gmail, https://www. boomeranggmail.com/; Mail Merge, https:/chrome.google. com/webstore/detail/mail-merge-for-g mail/ hcdbcahipmenkfgfpfmiodkncfkeannn?hl=en) that can be downloaded that create functionality like what is described. However, these other options are usually dependent on a third party or operate as a subscription-based service. This tutorial avoids these extraneous programs and asks the reader to engage in a little extra response effort for potentially more security and less cost.

The sample language used in the tutorial does not constitute legal advice. Organizations should consult with their attorneys and adjust this language to reflect the current health recommendations from trusted medical professionals. Each organization is responsible for ensuring that the language used within their own health attestation forms reflects their unique needs while ensuring compliance with all applicable laws (e.g., the Health Insurance Portability and Accountability Act [HIPAA], the Family Educational Rights and Privacy Act [FERPA]; U.S. Department of Education, 2020; U.S. Department of Health and Human Services, 2020). Finally, the health attestation system described herein will not work as intended if it is not supported by actionable policies and procedures. For example, the organization may specify that health attestations must be filled out by a certain time each day or within an hour of receiving or delivering services. Likewise, the organization may adopt a policy that specifies who is responsible for checking and following up with staff or consumers who fail to fill out their forms or indicate exposure to COVID-19. Finally, an organization would need to develop policies around how it should respond when a COVID-19 exposure is indicated on the attestation form. If a health attestation system is adopted by an organization, it should only be considered as one potential tool that may help mitigate the spread of COVID-19.

This tutorial assumes the organization already has a G Suite Basic, Business, or Enterprise subscription and that the reader has full administrative access to the Google Forms, Google Sheets, and Google Apps Script applications used to create the automated health attestation system. It is strongly recommended that readers create and maintain the automated health attestation system described under a designated organizational email account (e.g., attestation@organization.com). Likewise, the roles that organizational staff should adopt in managing the system should be clearly specified. For example, the organization's legal team could help draft the health attestation language used in the form. Additionally, the organization's human resources department and executive team could work together to develop policies for running the system. Finally, the organization's scheduling department could be responsible for receiving submitted attestation forms when COVID-19 exposure is identified so they can respond quickly.

\section{Creating a Health Attestation Form}

The following steps describe how to create a health attestation form using Google Forms. This tutorial is only designed to cover the functionality for establishing a basic health attestation system and does not describe unrelated steps such as theming the form to match an organization's branding.

1. Direct a browser to the https://docs.google.com/forms website.

2. Log in using the appropriate Google or G Suite account credentials if prompted.

3. If prompted, click the "Blank" option to open a new form. Otherwise, a new blank form will automatically open.

4. Click on the "Untitled form" text box located toward the center of the screen and type "COVID-19 Health Attestation Form" in the space provided.

5. Click on the "Form description" text box located directly under the form title and type the following statement: "The organization reserves the right and sole discretion to temporarily discontinue work if the following health attestation form is not filled out when required, if your form indicates that you or those you are in direct contact with are at risk of a COVID-19 infection, or if you do not acknowledge and agree with all of the compliance and risk acknowledgment statements below."

6. Click once on the "Untitled form" form name located in the top-left corner of the screen to automatically change the form name to the form title entered earlier.

7. Click the "Untitled Question" text box and type "Health Assessment" in the space provided. By default, the question type should be set to "Multiple Choice."

8. Click on the "Option 1" text box and type the following statement: "I hereby acknowledge that within the last 14 days, I and the people I remain in close physical contact with have not knowingly been within 6 feet of someone who has a laboratory-confirmed COVID-19 diagnosis OR experienced a fever, cough, difficulty breathing, 
F COVID-19 Health Attestation $\mathrm{F} \times+$

$\leftarrow \rightarrow \mathrm{C}$ a docs.google.com/forms/d/1stPqSqyZoaWgpfMux4cXFyfnn3v8bCIDCE2R1NNZRzg/e... 수응 incognito (3)

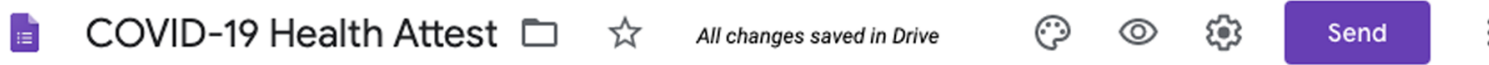

Questions Responses 44

\section{COVID-19 Health Attestation Form}

The organization reserves the right and sole discretion to temporarily discontinue work if the following health attestation form is not filled out when required, if your form indicates that you or those you are in direct contact with are at risk of a COVID-19 infection, or if you do not acknowledge and agree with all of the compliance and risk acknowledgement statements below.

\section{Email address *}

Valid email address

This form is collecting email addresses. Change settings

\section{Health Assessment}

I hereby acknowledge that within the last 14 days, I and the people I remain in close physical contact with, ...

\section{Compliance Acknowledgement}

I hereby acknowledge my compliance with the policies and procedures of the organization as the same m...

\section{Risk Acknowledgement}

I acknowledge that people with certain conditions are recognized by the Centers for Disease Control (CDC)...

$$
:::
$$

Health Attestation

$$
=\text { Short answer }
$$

By submitting this form, I hereby represent and warrant that (a) all information I provided herein is accurate and true as of the date hereof; (b) I completed this attestation at the request of the organization, and (c) my full name is:

Short answer text

而 Required $\bigcirc$ :

Figure 1 Screenshot Showing a Sample COVID-19 Health Attestation Form in Google Forms 
chills, muscle pain, headaches, sore throat, and/or new loss of taste or smell."

9. Click on the circled " + " button located to the right of the question to add another question.

10. Type "Compliance Acknowledgment" in the question text box.

11. Click "Option 1" text box and type the following statement: "I hereby acknowledge my compliance with the policies and procedures of the organization as the same may be updated from time to time, including those related specifically to addressing coronavirus (COVID-19) and the prevention thereof."

12. Click on the circled " + " button located to the right of the question to add another question.

13. Type "Risk Acknowledgment" in the question text box.

14. Click "Option 1" text box and type the following statement: "I acknowledge that people with certain conditions are recognized by the Centers for Disease Control (CDC) as being at a higher risk for COVID-19. To the extent any of these conditions apply to me, I agree to take all appropriate extra precautions including any such precautions advised by my licensed physician."

15. Click on the circled "+" button located to the right of the question to add the final question.

16. Type "Health Attestation" in the question text box.

17. Click on the "Multiple choice" drop-down menu and select the "Short answer" option to change the question format.

18. Click the switch located next to the "Required" option to make the question nonskippable.

19. Click the three vertical dots located to the right of the "Required" option.

20. Click the "Description" option.

21. Click the "Description" text box and type the following statement: "By submitting this form, I hereby represent and warrant that (a) all information I provided herein is accurate and true as of the date hereof, (b) I completed this attestation at the request of the organization, and (c) my full name is:"

22. Click the gear icon located in the top-right corner of the screen.

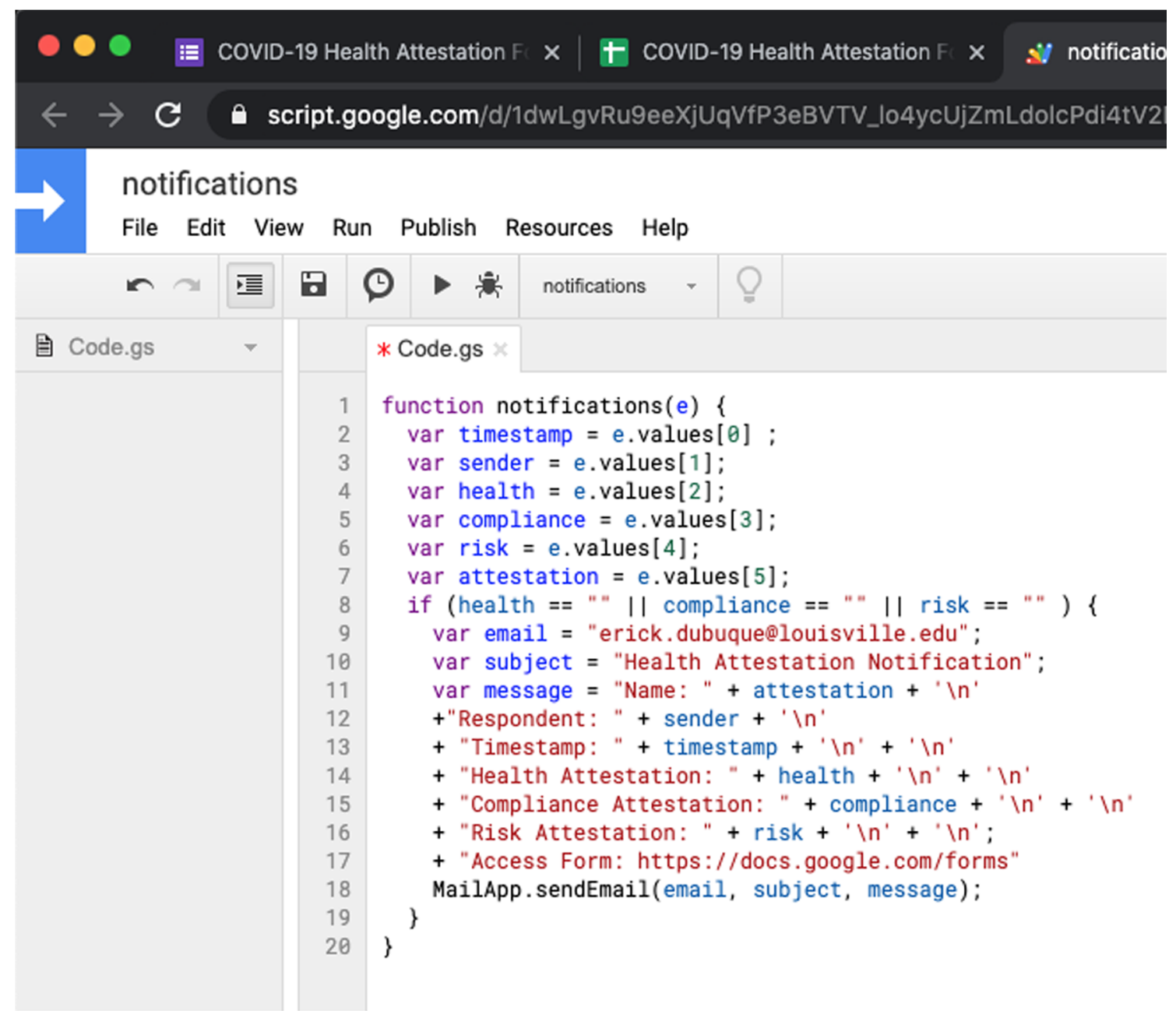

Figure 2 Screenshot of Code Used in Google Apps Script to Automate Notifications When Health Attestations Are Submitted in Google Forms 


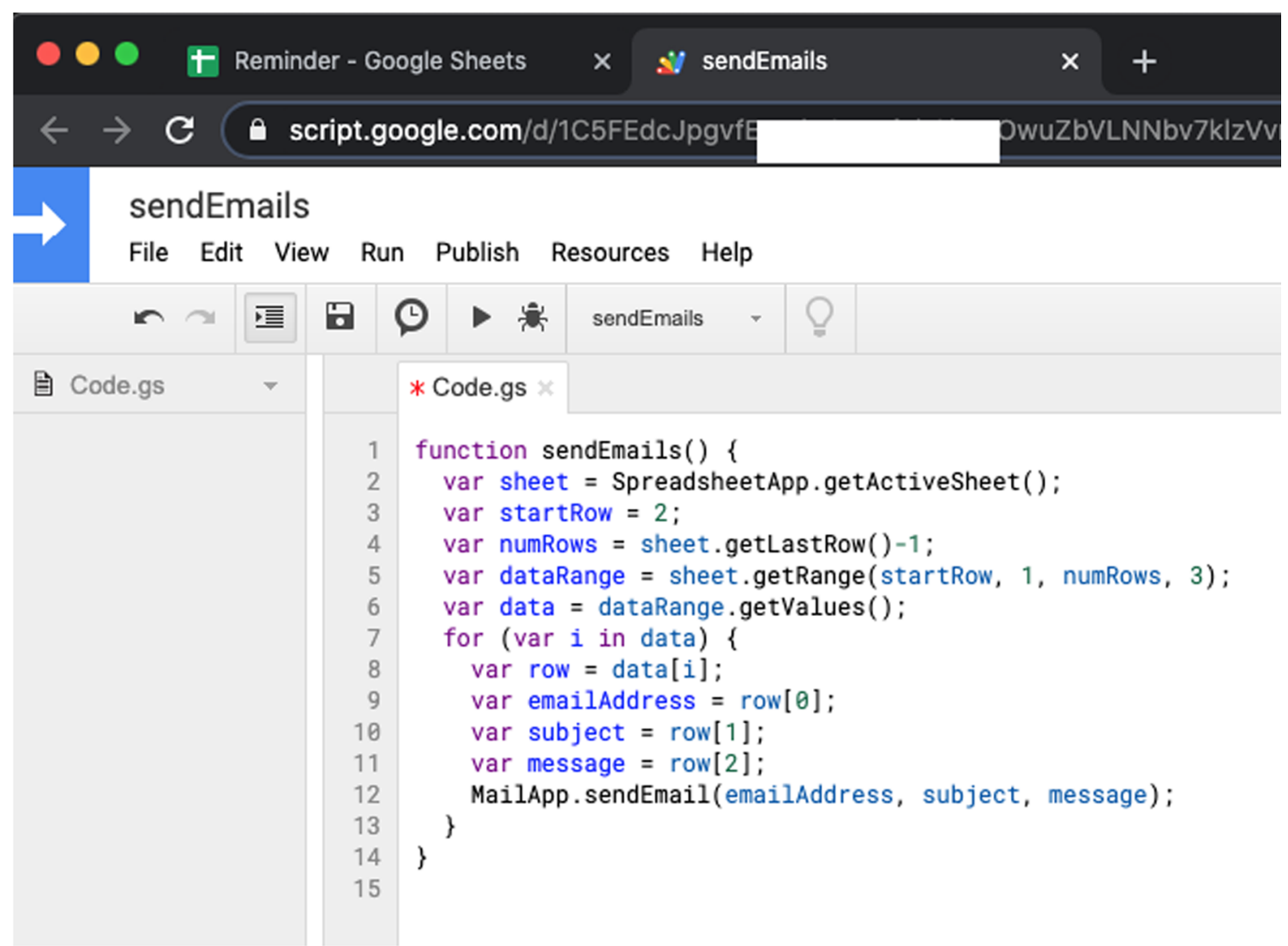

Figure 3 Screenshot of Code Used in Google Apps Script to Automate Reminder Emails to Potential Respondents to Prompt Completion of Health Attestation Forms

23. Check the "Collect email addresses" option to automatically add a question to the form that requests an email address.

24. Check the "Response receipts" option to give respondents an option to receive an emailed copy of their submission. The default setting provides the respondent with an opportunity to receive a copy of their submission, but it can be set to "Always" if preferred.

25. If creating the form within a G Suite account, uncheck the "Restrict to users in [organization name] and its trusted organizations" option to allow anyone to access the form. This option is not available on personal Google accounts.

26. Click the "Save" button.

27. Click the "Send" button located in the top-right corner of the screen.

28. Click the link icon located between the envelope and chevron $(<>)$ icons.

29. Click the "Shorten URL" checkbox to create a shorter address link to the form.

30. Click the "Copy" button to copy the link.

31. Paste the hyperlink in a safe place, as it will be used later to share access to the form with potential respondents.

32. Click the " $\mathrm{X}$ " button to close the pop-up window.

\section{Customizing Email Notifications}

Automated email notifications are an excellent way to quickly inform the organization when potential exposure to COVID-19 has been indicated on a health attestation form submission. These notifications can be sent to designated individuals as soon as the form is submitted allowing for a quick response (e.g., cancel the appointment, start contact tracing). In this section, the steps for creating customized notifications when respondents submit health attestation forms will be described. Normally, setting notifications when respondents submit forms is straightforward using Google Forms. However, for the health attestation system to manage large groups of respondents, notifications should only be sent when a respondent submits a health attestation form that requires follow-up. In other words, all form submissions should be time-stamped and logged, but the only time an administrator should be notified by email is when a submission indicates exposure to COVID-19 or failure to comply with policies or acknowledge risk. This allows an organization to respond quickly when necessary without having to search through multiple email notifications. 
1. Direct a browser to the https://docs.google.com/forms website. $^{1}$

2. Log in using the appropriate G Suite account credentials if prompted.

3. Click the health attestation form created earlier.

4. Click the "Responses" tab located in the top middle of the webpage.

5. Click the green spreadsheet icon located in the upperright corner of the screen above the "Accepting responses" option.

6. Click the "Create" button when the pop-up window appears to create a new spreadsheet.

7. Click "Tools," then "Script Editor" in the top menu bar to open a new tab in the browser.

8. Click the "Untitled project" text box located in the topleft corner.

9. Type "notifications" in the "Edit Project Name" pop-up box.

10. Click the "OK" button.

11. Clear the main code window by highlighting and deleting the following text:

\section{function myFunction() \{}

$$
\text { \} }
$$

12. Enter the following replacement code into the main code window: ${ }^{2}$

13. Within the code from the previous step, replace the "INSERT EMAIL(S) HERE" text with the email addresses of anyone that should receive the notification. The email addresses must remain within quotation marks. Multiple email addresses should be separated by a comma. For example:

var email="attestation@organization.com, scheduler@billing.com”;

14. Click "File," then "Save" in the top menu bar.

15. Click "Edit," then "Current project's trigger" in the top menu bar to open a new tab in the browser.

16. Click the "+ Add Trigger" button located in the bottomright corner of the screen.

17. Under the "Select event type" header, click the "On form submit" option from the drop-down list.

18. Click the "Save" button.

19. Click the G Suite email address connected to the account.

20. If the "This app isn't verified" message appears, click the "Advanced" link.

\footnotetext{
${ }^{1}$ Readers continuing the tutorial from the previous section may begin at Step 4.

${ }^{2}$ In the interest of brevity, a full explanation of each line of code is not provided. However, more information about creating these steps can be found within the Google Apps Script Guide (Google, 2020a, 2020b).
}

21. Click the "Go to notifications (unsafe)" link.

22. Click the "Allow" button.

\section{Sending Automated Reminder Emails in App Script}

The following steps describe how to set up automated email reminders for respondents expected to fill out the health attestation forms on a regular basis. For example, these steps can be used to automatically send reminders to employees or recipients of services every morning between 6:00 a.m. and 7:00 a.m.

1. Direct a browser to the https://docs.google.com/ spreadsheets website.

2. Log in using the appropriate G Suite account credentials if prompted.

3. Click the "Blank" option located under the "Start a new spreadsheet" header.

4. Click on the "Untitled spreadsheet" text box located in the top-left corner.

5. Type "Reminder" in the text box.

6. Click cell A1 and type "Email."

7. Starting in cell $\mathrm{A} 2$, enter the email addresses of the individuals who should receive the reminder message. Email addresses entered into the cell should be separated by commas (e.g., janedoe@gmail.com, smith@outlook.com, mohammad@gmail.com). ${ }^{3}$

8. Click cell B1 and type "Subject."

9. Click cell B2 and type "Health Attestation Reminder."

10. Click cell $\mathrm{C} 1$ and type "Message."

11. Click cell $\mathrm{C} 2$ and type "This is a reminder for you to fill out the health attestation form found at" followed by a link to the health attestation form created earlier.

12. Click "Tools," then "Script Editor" in the top menu bar to open a new tab in the browser.

13. Click the "Untitled project" text box located in the topleft corner.

14. Type "sendEmails" in the "Edit Project Name" pop-up box.

15. Click the "OK" button.

16. Clear the main code window by highlighting and deleting the following text:

\section{function myFunction() \{}

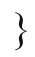

\footnotetext{
${ }^{3}$ Email addresses will need to be carefully cultivated when using the system. There should be a system in place to add and remove these addresses in the Google Sheet if an employee or consumer leaves or joins the organization.
} 
17. Enter the following replacement code into the main code window:

18. Click "File," then "Save" in the top menu bar.

19. Click "Edit," then "Current project's trigger" in the top menu bar to open a new tab in the browser.

20. Click the " + Add Trigger" button located in the bottomright corner of the screen.
21. Under the "Select event source" header, click the "Timedriven" option from the drop-down list.

22. Under the "Select type of time based trigger" header, click the "Day timer" option or choose a different option that reflects how frequently you would like respondents to be reminded to fill out the health attestation form.

$$
\begin{aligned}
& \text { function notifications(e) }\{ \\
& \text { var timestamp }=\text { e.values }[0] \text {; } \\
& \text { var sender }=\text { e.values[1]; } \\
& \text { var health }=\text { e.values[2]; } \\
& \text { var compliance }=\text { e.values }[3] \text {; } \\
& \text { var risk }=\text { e.values }[4] \text {; } \\
& \text { var attestation }=\text { e.values }[5] \text {; } \\
& \text { if }(\text { health }==\text { "' }|| \text { compliance }==\text { "' || risk }==\text { "" })\{ \\
& \text { var email = "INSERT EMAIL(S) HERE"; } \\
& \text { var subject = "Health Attestation Notification"; } \\
& \text { var message }=\text { "Name: " + attestation }+ \text { "In' } \\
& \text { +"Respondent: " + sender + ' 'n' } \\
& \text { + "Timestamp: " + timestamp + ' } \mathrm{n} \text { ' + 'In' } \\
& \text { + "Health Attestation: " + health + 'In' + '\n' } \\
& \text { + "Compliance Attestation: " + compliance + ' In' + ' } n \text { ' } \\
& \text { + "Risk Attestation: " + risk + ' 'n' + 'ไn'; } \\
& \text { + "Access Form: https://docs.google.com/forms" } \\
& \text { MailApp.sendEmail(email, subject, message); } \\
& \text { \} }
\end{aligned}
$$




\section{function sendEmails ()\{}

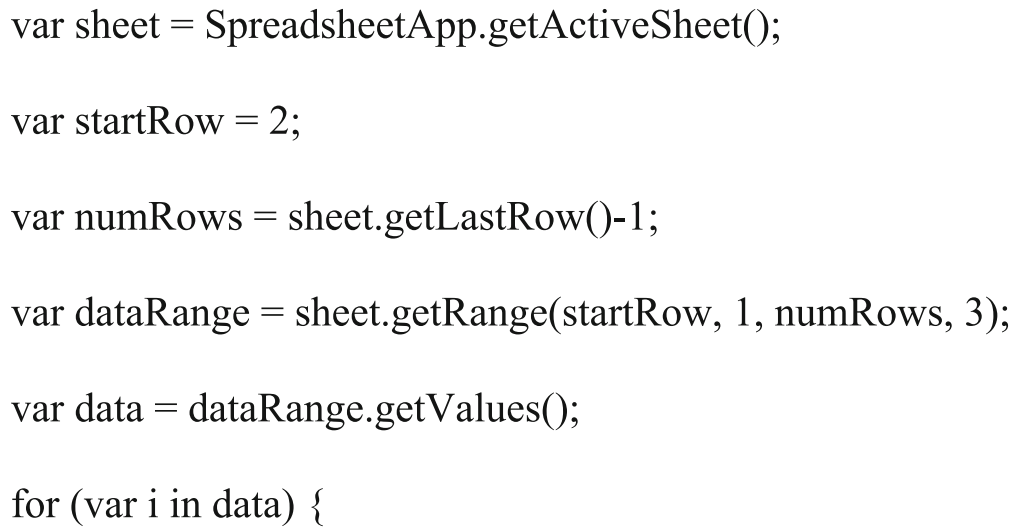

var row $=\operatorname{data}[i]$

var emailAddress $=\operatorname{row}[0]$;

$\operatorname{var}$ subject $=$ row $[1]$;

var message $=$ row $[2]$;

MailApp.sendEmail(emailAddress, subject, message);

\}

\}

23. Under the "Select time of day" header, click the "6am to $7 \mathrm{am}$ " option or select a different time frame when you would like the reminder email sent out.

24. Click the "Save" button.

25. Click the G Suite email address connected to the account.

26. If the "This app isn't verified" message appears, click the "Advanced" link.

27. Click the "Go to sendEmails (unsafe)" link.

28. Click the "Allow" button.

\section{Discussion}

The experience of owning, managing, and consulting with a variety of behavior-analytic clinics, schools, and businesses gives us broad vantage to propose a solution that should help across many different contexts. Specifically, it is important to recognize the value in customizing an organization's attestations and to have a system controlled by the entity. This importance is especially apparent during the pandemic when regulatory recommendations, guidelines, and requirements change. Organizations must flexibly and rapidly adapt to such changes while maintaining required HIPAA, FERPA, and other confidentiality needs. Although attestations are but one part of the solution, the following examples will illustrate a variety of ways in which they can be helpful.

Consider a variety of cases in which this system may be used. In the case of home-based care, the organization may require that an attestation must be completed by both parties (staff and consumer) before a session can commence. If the organization delivers two sessions per day, this might require a total of three attestations. Failure to get any one of those may create service barriers and delays on a frequent basis. Likewise, in a center-based program, the process used for collecting attestations may differ. The consumer attestation can occur at drop-off and can be accomplished with a center-owned device and internet connection. Additionally, staff members can attest at drop-off, before they enter, but while still on site. Although there may be multiple sessions per day, the number of attestations may be reduced because 
the environment is more controlled, and the influx of new people is limited. Finally, a school-based setting may share similarities with the center-based therapy settings, except that sometimes the drop-off and pickup are done via transportation. This means that if third-party transportation drops off a learner, and an attestation has not been completed, a problematic situation arises. In such situations, it will be important to have a plan of action ready to avoid such risks and vulnerabilities.

In conclusion, this tutorial provides a low-cost option for small businesses, but one that is also equally accessible and scalable as organization size increases. As with all solutions, there are limitations, as noted previously, and once understood, the attestation process is adjusted for each instance. Foremost is that any use of electronic technology and webbased solutions means that all participants will need access to electronic hardware (computers or mobile devices) and the internet. If this technology is not readily available to all members of an organization, it will limit the usability of the system, potentially leaving gaps in the protection afforded by the attestation in the first place. For instance, if some of the consumers or employees do not know how to complete the forms, and the system operator makes occasional allowances, these vulnerabilities can defeat the purpose (precluding infection spread). For an organization, this means that although you may have access to free software, and this tutorial provides the means to develop an attestation system, the organization still has some work to do. Specifically, there need to be systems of training and compliance to make sure that all who need to attest have the access, tools, and knowledge to implement and submit their attestations. Second, there will need to be a system that compares attestation submissions with expectations and flags any discrepancies for review and intervention. If, for instance, an organization creates an attestation requirement prior to session delivery on a daily basis, there needs to be a system to enforce the requirement, or the organization is simply creating a sense of false security for all the members and potential legal issues in terms of health injury and potential discrimination litigation.

Finally, the sample language used does not provide any legal or health protection; rather, the purpose of this article was to describe a technology that can be used to deliver communication and receive input. The advantage of this limitation, however, is that once an organization has used this technology, they may find that other problems can be solved using a similar automated communication system.

Acknowledgements The authors would like to acknowledge Emily Zoernig for her assistance in piloting and providing feedback on the tutorial.

\section{Declarations}

Funding No funding was provided for this project.

Conflict of interest The authors declare that there is no conflict of interest.

Ethical approval This project was submitted to the University of Louisville Human Subjects Protection Program and was deemed to not meet the "Common Rule" definition of human subjects research. As such the project did not require institutional review board approval.

Informed consent This project did not utilize any human subjects and relied on publicly available data sets.

\section{References}

Dubuque, E. M., Yingling, M. E., Ranade, E. S., \& Dubuque, M. L. (2020). Using health attestation forms to protect organizations, employees, and clients during the COVID-19 global pandemic. Behavior Analysis in Practice. Advance online publication. https:// doi.org/10.1007/s40617-020-00495-y

Google. (2020a, August 31). Tutorial: Sending emails from a spreadsheet. https://developers.google.com/apps-script/articles/ sending_emails

Google. (2020b, August 31). Simple triggers. https://developers.google. com/apps-script/guides/triggers

Gostic, K., Gomez, A. C., Mummah, R. O., Kucharski, A. J., \& LloydSmith, J. O. (2020). Estimated effectiveness of the symptom and risk screening to prevent the spread of COVID-19. eLife, 9, Article e55570. https://doi.org/10.7554/eLife.55570.

Güner, R., Hasanoğlu, I., \& Aktaş, F. (2020). COVID-19: Prevention and control measures in community. Turkish Journal of Medical Sciences, 50(SI-1), 571-577. https://doi.org/10.3906/sag-2004-146.

Hur, J., \& Cheol Chang, M. (2020). Usefulness of an online preliminary questionnaire under the COVID-19 pandemic. Journal of Medical Systems, 44(166), 1-2. https://doi.org/10.1007/s10916020-01586-7.

Jeong, H., Lee, J., Kim, J., Choen, S., Sohn, K. M., Kim, Y., \& Kiem, S. (2020). Self-assessment questionnaire for efficient and safe evaluation of patients with mild COVID-19. Infection and Chemotherapy, 52(2), 212-215. https://doi.org/10.3947/ic.2020.52.2.212.

Johns Hopkins University. (2020, October 19). COVID-19 dashboard by the Center for Systems Science and Engineering (CSSE) at Johns Hopkins University (JHU). https://coronavirus.jhu.edu/map.html

Torales, J., O’Higgins, M., Castaldelli-Maia, J. M., \& Ventriglio, A. (2020). The outbreak of COVID-19 coronavirus and its impact on global mental health. International Journal of Social Psychiatry. https://doi.org/10.1177/0020764020915212

U.S. Department of Education. (2020, March). FERPA and the coronavirus disease 2019 (COVID-19). https://studentprivacy.ed.gov/ resources/ferpa-and-coronavirus-disease-2019-covid-19

U.S. Department of Health and Human Services. (2020, August 31). HIPAA and COVID-19. https://www.hhs.gov/hipaa/forprofessionals/special-topics/hipaa-covid19/index.html

Publisher's Note Springer Nature remains neutral with regard to jurisdictional claims in published maps and institutional affiliations. 